\title{
Statistical Simulation of Thin-Film Bearings
}

\author{
Jian-Zheng Jiang, Ching Shen, and Jing Fan \\ Laboratory of High Temperature Gas Dynamics, Institute of Mechanics, CAS, Beijing 100080, China
}

\begin{abstract}
The information preservation (IP) method and the direct simulation Monte Carlo (DSMC) method are used to simulate the gas flows between the write/read head and the platter of the disk drive (the slider bearing problem). The results of both methods are in good agreement with numerical solution of the Reynolds equation in the cases studied. However, the DSMC method owing to the problem of large sample size demand and the difficulty in regulating boundary conditions at the inlet and outlet was able to simulate only short bearings, while IP simulates the bearing of authentic length $\sim 1000 \mu \mathrm{m}$ and can provide more detailed flow information.
\end{abstract}

\section{INTRODUCTION}

The study of ultra thin gas film bearings has become of great interest in recent years. In a modern Winchestertype disk drive, the write/read head floats approximately $50 \mathrm{~nm}$ above the surface of the spinning platter ${ }^{[1,2]}$. The head and platter together with the gas layer in between form a slider air bearing. The prediction of the vertical force on the head (as obtained from the pressure distribution in the gas) is a crucial design premise.

Traditionally, Reynolds equation has been used to model slider bearings ${ }^{[3]}$. Fukui and Kaneko showed that the solution of the linearized Boltzmann equation for the thin film bearing problem can be decomposed into the solutions of the plane Couette and the Poiseuille flows and derived the generalized Reynolds equation ${ }^{[4]}$.

Because the characteristic length scale of thin-film bearings, i.e. the height between the head and platter $h$, is comparable with the mean free path of molecules, the Knudsen number $(K n=\lambda / h)$ is no longer small enough to be neglected. Molecular-based numerical schemes, such as the direct simulation Monte Carlo (DSMC) method ${ }^{[5]}$, are physically appropriate for this kind of gas flows where non-continuum, rarefied gas effects become important. Alexander et al. have simulated the short thin-film bearings using the DSMC method ${ }^{[6]}$. However, because of the statistical scatter inherent in the DSMC method and the difficulty in regulating boundary conditions at the inlet and outlet the DSMC simulation was done only for short head problem.

Meanwhile the information preservation (IP) method ${ }^{[7,8]}$ emerged as an alternative numerical technique to analyze subsonic, micro-scale gas flows. It has been successfully used to simulate some gas flows in micro-electromechanical-systems (MEMS) ${ }^{[9-15]}$. Shen et al. ${ }^{[9]}$ by employing the measures of the conservation scheme of the mass conservation and the super-relaxation method calculated the gas flows in long micro-channels with results in good agreement with the experimental data ${ }^{[16,17]}$.

In this paper, we present the results obtained by the IP method of simulation of the slider bearing problem and compare them with the DSMC calculations and the solutions of Reynolds equation. Firstly, the Reynolds equation for the slider problem and the IP method are briefly reviewed. Then we present and compare the results of the three methods. Finally some concluding remarks are given.

\section{REYNOLDS EQUATION}

The basic geometry of the two-dimensional gas flow scheme in the gas film bearing problem is outlined in Fig. 1 . The upper boundary is a stationary, slightly inclined surface, and the lower boundary moves to the right with velocity $U$. The gas and the surfaces are assumed to be at a constant, ambient temperature $T_{0}$. Since the incline is

CP762, Rarefied Gas Dynamics: 24 $4^{\text {th }}$ International Symposium, edited by M. Capitelli (C) 2005 American Institute of Physics 0-7354-0247-7/05/\$22.50 
slight, typically less than $1^{\circ}$, the pressure $p$ is taken to be constant in the vertical direction. At the left $(x=0)$ and right $(x=L)$ boundaries the pressure in the gas is fixed at ambient pressure $p_{0}$.

From standard lubrication theory ${ }^{[3]}$ in the continuum regime the pressure in the gas may be computed from the Reynolds equation

$$
\frac{d}{d x}\left(p h^{3} \frac{d p}{d x}\right)=6 \mu U \frac{d}{d x}(p h),
$$

where $\mu$ is the viscosity of the gas, and $h(x)$ is the vertical spacing between the walls. Its nondimensional form is

$$
\frac{d}{d X}\left(P H^{3} \frac{d P}{d X}\right)=\Lambda \frac{d}{d X}(P H)
$$

where $X=x / L, P=p / p_{0}, H=h / h_{0}, h_{0}=h(x=L)$ is the minimum spacing between the walls, and the bearing number is defined as $\Lambda=6 \mu U L /\left(p_{0} h_{0}^{2}\right)$. Burgdorfer first introduced the Reynolds equation with slip correction ${ }^{[18]}$,

$$
\frac{d}{d X}\left(\left[1+\frac{12-6 \sigma}{\sigma} K n\right] P H^{3} \frac{d P}{d X}\right)=\Lambda \frac{d}{d X}(P H),
$$

where $\sigma$ is the accommodation coefficient of the walls and $K n=\lambda / h$ is the local Knudsen number. Fukui and Kaneko showed that the solution of the linearized Boltzmann equation for the thin film bearing problem can be decomposed into the solutions of the plane Couette and the Poiseuille flows and derived the generalized Reynolds equation ${ }^{[4]}$

$$
\frac{d}{d X}\left[\bar{Q}_{p}(K n) P H^{3} \frac{d P}{d X}\right]=\Lambda \frac{d}{d X}(P H) .
$$

The function $\bar{Q}_{p}(K n)$ is nondimensional flow rate for Poiseuille flow; a database of tabulated values for $\bar{Q}_{p}(K n)$ is given in Ref. [19]. For $\sigma=1$, Robert gives the fitting formula ${ }^{[4]}$

$$
\bar{Q}_{p}(K n) \cong 1+6 A K n+\frac{12}{\pi} K n \log (1+B K n),
$$

where the parameters $A=1.318889$ and $B=0.387361$ are selected so as to give a best fit to the data in Ref. [19]. The data presented in [19] and expressed by formula (5) are drawn in Fig2, from which it is seen the above expression for $\bar{Q}_{p}(K n)$ is in good agreement with the data of Ref. [19], and it can be used to obtain the numerical results of the generalized Reynolds equation (4) for arbitrary Knudsen number when $\sigma=1$.

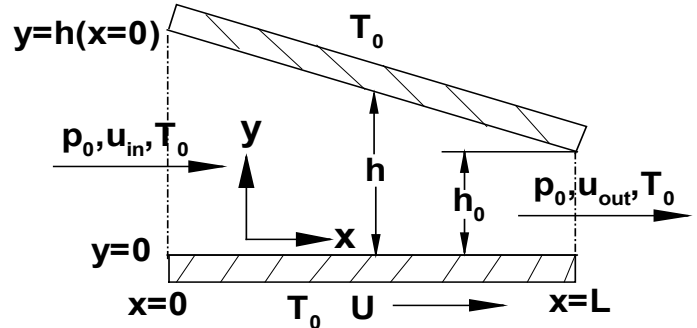

FIGURE 1. Schematic diagram of the slider bearing

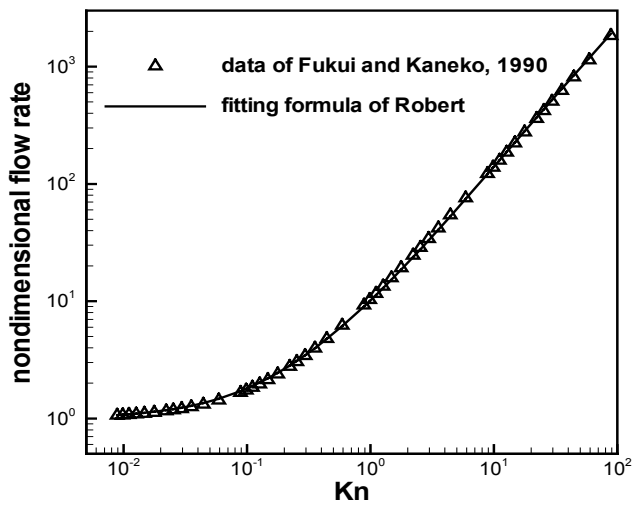

FIGURE 2. Relation of the nondimensional flow rate for Poiseuille flow versus Knudsen number for the case $\sigma=1$ 


\section{THE IP METHOD}

Because the statistical fluctuation in the DSMC method becomes insurmountably large at the low Mach numbers that are characteristic of MEMS, where the low macroscopic speed causes a large noise to useful information ratio, and a very large sampling size is demanded, the information preservation (IP) method is proposed to reduce the statistical fluctuation ${ }^{[7,8]}$. The IP method was successfully applied to a number of problems ${ }^{[9-15]}$. Implementation of the IP method has been described in detail in these references and will be omitted here with only some experiences in this particular problem elucidated.

The rectangular area (from $x=0$ to $x=L$, and from $y=0$ to $y=h(x=0)$ ) is divided into uniform rectangular cells. Some of them are incised by upper surface into two parts, only the one under the upper surface is within the computational domain. The cells of this part are called incomplete cells. The smallest volume of the incomplete cells is much less than that of the standard complete cell. A measure is undertaken that each incomplete cell is combined with its lower adjacent complete cell to avoid extreme small number of simulated molecules in a cell.

Meanwhile, a smoothing procedure is used to eliminate the no-physical oscillation. Here, a simple weighted average method is used.

$$
\rho_{i, j}^{\prime}=c_{1} \rho_{i, j}+\left(1.0-c_{1}\right) \times\left(\rho_{i-1, j}+\rho_{i, j-1}+\rho_{i+1, j}+\rho_{i, j+1}\right) / 4
$$

where, the superscript " '" denotes the value after smoothing, and $c_{1}$ is the weighting factor. The value of $c_{1}$ denotes the proportion the newly attained value occupies in the value after smoothing. If $c_{1}$ trends to 1 , there is not any smoothing measure undertaken. The second term on the right hand side of equation (6) shows the effect of the value of adjacent cells. Because the temperature variation in the flows is neglected, as long as the density is smoothed, the pressure is also smoothed. The concrete value of $c_{1}$ used in simulation will be specified separately.

The ambient pressure is used as the pressure boundary condition, it can be used to calculate the acceleration of the gas in the boundary cells. The mass flux traversing the cross-sections at the central line of the boundary cells is used as the mass flux at the boundary, and is used to calculate the density increment of the gas in the boundary cells.

The super-relaxation scheme, such as suggested in Ref. [9], is also employed here to accelerate the convergence of the process

$$
\rho_{i, j}^{t+\Delta t}=\rho_{i, j}^{t}+\omega \Delta \rho_{i, j}^{t}
$$

The concrete value of $\omega$ used in simulation will be specified separately in each case later.

\section{RESULTS}

A series of slider bearing were studied. For all runs the ratio $h(x=0) / h_{0}$ of the entrance to exit heights is 2 , and the exit height is $h_{0}=50 \mathrm{~nm}$. The ambient pressure $p_{0}$ is the atmospheric pressure, and the ambient temperature is $T_{0}=273 \mathrm{~K}$. The viscosity of the gas is $\mu=2.08 \times 10^{-5} \mathrm{Ns} / \mathrm{m}^{2}$. The lower platter speed is $U=25 \mathrm{~m} / \mathrm{s}$. For the DSMC code and the IP calculation, the hard sphere (HS) molecular model is used to simulate the interaction between the molecules. For the first three cases, the solution of the Reynolds equation (4) is used as the initial pressure distribution of both IP and DSMC calculation, and the initial velocity is zero.

The Reynolds equation (4) is solved numerically only for the case of $\sigma=1$, when the Poiseuille flow rate given by equation (5) can be used. The Reynolds equation is integrated by using the targeting method to obtain the pressure distribution of the slider bearings.

First we consider a system of length $L=5 \mu \mathrm{m}$. The accommodation coefficient of the walls is $\sigma=1$. The pressure distribution obtained by the IP and DSMC methods and the solution of the Reynolds equation (4) together with the results of the DSMC method given by Alexander et al. are presented in Fig. 3. All data are in good agreement with each other. Fig. 4 shows the velocity component in the $\mathrm{X}$-direction along the vertical line at different $\mathrm{X}(\mathrm{X}=0.2,0.4$, $0.6,0.8,0.9)$ positions obtained by the IP method and the DSMC method. They agree well with each other. From Fig. 4 it is seen that, the velocity profiles at $X=0.2$ and $X=0.4$ are concave and the velocity profile at $X=0.9$ is convex, because the pressure gradient at $X=0.2$ and $X=0.4$ is negative, and that at $X=0.9$ is positive. And because of microscopic flow structure, the velocity profiles of Couette flows are no longer linear, they become convex near the upper surface and concave near the lower platter. The velocity profiles obtained from the overlying of Couette and Poiseuille flows at the two other places are not purely concave or convex. The time step of the IP method is about 
$0.012 \tau$ ( $\tau$ is the collision time). The weighting factor $c_{1}$ in smoothing code is 0.9996 and the maximum superrelaxation factor $\omega$ in equation (7) is 15 . Both the IP and DSMC method begin sampling after $2 \times 10^{4}$ time steps. The sample size of the IP method is about $2 \times 10^{5}$ and that of the DSMC method is about $2 \times 10^{6}$.

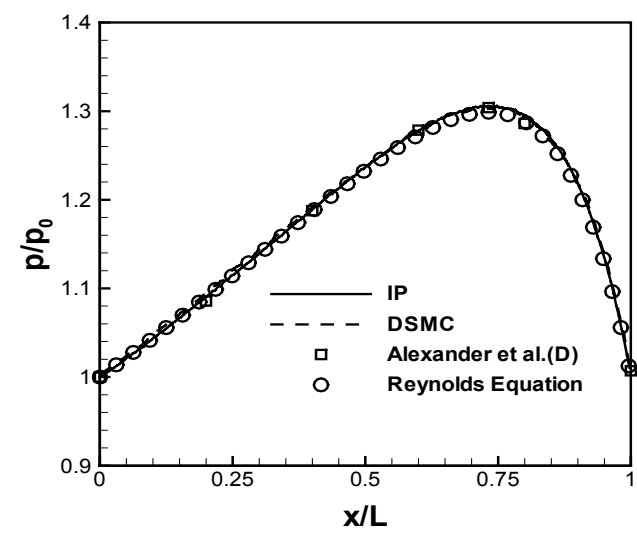

FIGURE 3. The pressure distribution of the slider bearing for $L=5 \mu \mathrm{m}$ and $\sigma=1$

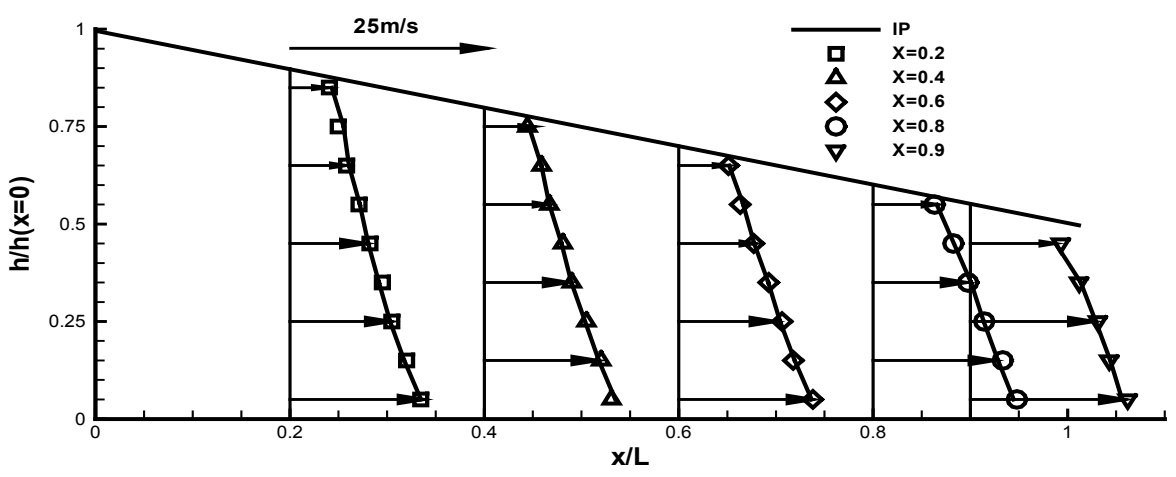

FIGURE 4. The $\mathrm{x}$-direction velocity profiles at different $\mathrm{X}$ positions for $L=5 \mu \mathrm{m}$ and $\sigma=1$

Then slider bearing with $L=25 \mu \mathrm{m}$ is considered. The pressure distribution obtained by the IP method compared with the solution of the Reynolds equation (4) is shown in Fig.5. They agree well with each other. The time step of the IP method is about $0.06 \tau$. The weighting factor $c_{1}$ in smoothing code is 0.99992 and the super-relaxation factor $\omega$ of 5 is used. After $10^{4}$ time steps, sampling begins. The sample size of the IP method is about $10^{5}$.

As an example having direct application interest, we consider a slider bearing with $L=1000 \mu \mathrm{m}$, which is the typical authentic length of the head of the hard disk drives. The pressure distribution given by the IP method is also in good agreement with the solution of the Reynolds equation (4) (shown in Fig.6). The time step of the IP method is about $0.06 \tau$. The weighting factor $c_{1}$ in smoothing code is 0.999996 and the maximum super-relaxation factor $\omega$ in equation (7) is 5 . After $2 \times 10^{4}$ time steps, sampling begins. The sample size of the IP method is about $3 \times 10^{5}$.

Finally we consider a system of length $L=5 \mu \mathrm{m}$. However, The accommodation coefficient of the walls is $\sigma=0.7$. The pressure distribution of the system is shown in Fig.7. And the present results of the IP method and the DSMC method are in agreement with the data given by Alexander et al. for the solution of the Reynolds equation (4). 


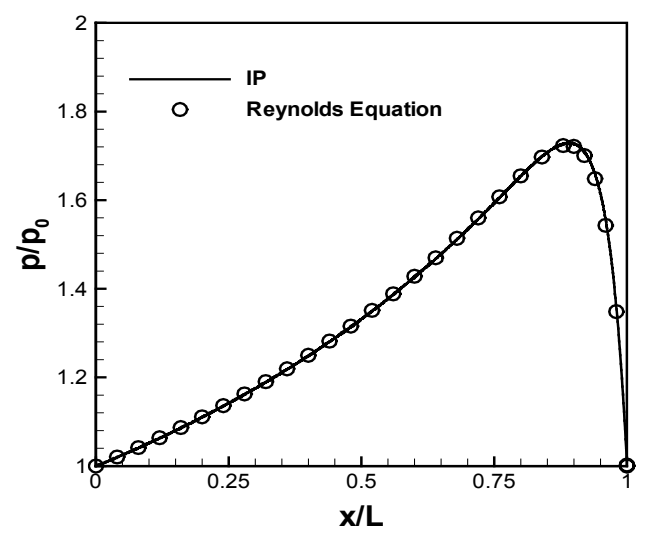

FIGURE 5. The pressure distribution of the slider bearing for $L=25 \mu \mathrm{m}$ and $\sigma=1$

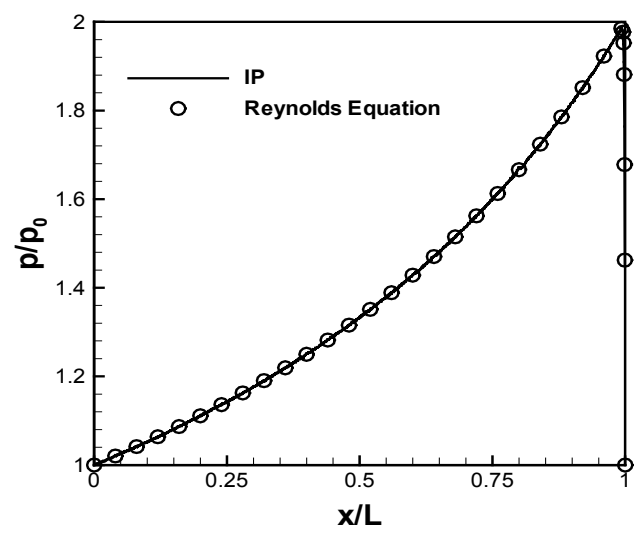

FIGURE 6. The pressure distribution of the slider bearing for $L=1000 \mu \mathrm{m}$ and $\sigma=1$

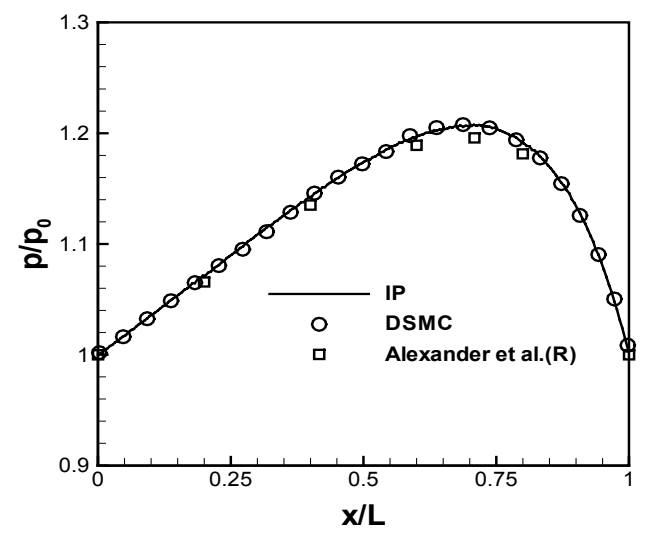

FIGURE 7. The pressure distribution of the slider bearing for $L=5 \mu \mathrm{m}$ and $\sigma=0.7$

\section{CONCLUSION}

The IP method can be used to simulate the ultra-thin gas film bearing problem including the case of authentic length and [0]wall with incomplete accommodation. The results obtained by the IP method are in good agreement with the calculation of the DSMC method and the solution of the Reynolds equation (4). The latter can be considered as a verification of the IP method by a touchstone of the merit of strict kinetic theory in simulating $2 \mathrm{D}$ microscale flows.

The number of the simulated molecules in a cell must not be too small, say, more than 10 molecules in a cell. The smoothing procedure with appropriate weighting factor (usually $>0.99$ ) can be used to eliminate the no-physical oscillation at the same time not to distort the pressure distribution, and the super-relaxation scheme can be employed to accelerate the convergence of the process.

\section{ACKNOWLEDGMENTS}

The authors appreciate support from NNSFC under Grant 90205024. 


\section{REFERENCES}

1. Deckert, K. L., "Computer-aided design of slider bearings in magnetic disk files”, IBM. J. Res. Devel. 35, 660 (1990).

2. Tagawa, N., "State of the art for flying head slider mechanisms in magnetic recording disk storage", Wear 168, 43 (1993).

3. Gross, W. A., Match, L. A., Castelli, V., Eshel, A., Vohr, J. H., and Wildmann, M., Fluid Film Lubrication (Wiley, New York, 1980).

4. Fukui, S., and Kaneko, R., "Analysis of ultrathin gas film lubrication based on linearized Boltzmann equation: First report derivation of a generalized lubrication equation including thermal creep flow", J. Tribology 110, 253 (1988).

5. Bird, G. A., Molecular Gas Dynamics and the Direct Simulation of Gas Flows, (Clarendon Press, Oxford, 1994).

6. Alexander, F. J., Garcia, A. L., and Alder, B. J., “ Direct simulation Monte Carlo for thin-film bearings”, Phys. Fluids 6 (12), 3854 (1994).

7. Fan, J., and Shen, C., "Statistical simulation of low-speed unidirectional flows in transition regime" in Rarefied Gas Dynamics, edited by R. Brun et al, Cepadues Editions, Vol. 2, 245 (1999).

8. Fan, J., and Shen, C., "Statistical simulation of low-speed rarefied gas", J. Comp. Phys. 167, 393 (2001).

9. Shen, C., Fan, J., and Xie, C., "Statistical simulation of rarefied gas flows in micro-channels", J. Comp. Phys. 189,512 (2003).

10. Cai, C. P., Boyd, I. D., Fan, J., and Candler G. V., "Direct simulation methods for low-speed microchannel flows",J. Thermophysics and Heat Transfe 14, 368 (2000).

11. Fan, J., Boyd, I. D., Cai, C. P., Hennighausen, K., and Candler G. V., " Computation of rarefied gas flows around a NACA 0012 airfoil", AIAA J. 39, 618 (2001).

12. Sun, Q., Boyd, I. D., and Candler, G. V., "Numerical simulation of gas flow over micro-scale airfoils", AIAA 2001-3071 (2001).

13. Sun, Q.H., and Boyd, I.D., “A Direct Simulation for Subsonic Microscale Gas Flows”, J. Comp. Phys. 179, 400 (2002).

14. Xie, C., Fan, J., and Shen, C., "Rarefied Gas Flows in Micro-Channels”, in Rarefied Gas Dynamics, ed. By Ketsdever and Muntz, 800 (2003).

15. Shen, C., Rarefied Gas Dynamics (in Chinese), National Defense Industry Press, Beijing (2003), English translation, Springe Verlag, to appear.

16. Pong, K. C., Ho, C. M., Liu, J. Q., and Tai, Y. C., "Non-linear pressure distribution in uniform micro-channels", ASMEFED 197, 51 (1994).

17. Shih, J. C., Ho, C. M., Liu, J. Q., and Tai, Y. C., "Monatomic and polyatomic gas flow through uniform microchannels", ASME-DSC 59, 197 (1996).

18. Burgdorfer, A., "The influence of the molecular mean-free path on the performance of hydrodynamic gas lubricated bearings", Trans. ASME 81, 94 (1959).

19. Fukui, S., and Kaneko, R., "A database for interpolation of Poiseuille flow rates for high Knudsen number lubrication problem", J. Tribology 112, 78 (1990). 